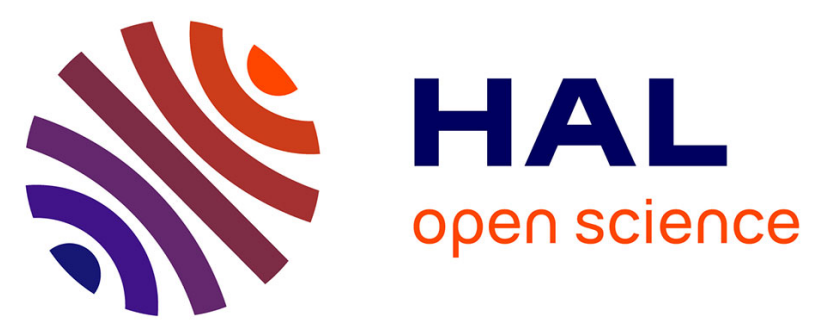

\title{
Homogenization of an elastic material reinforced by very strong fibers arranged along a periodic lattice
}

\author{
Houssam Abdoul-Anziz, Lukáš Jakabčin, Pierre Seppecher
}

\section{To cite this version:}

Houssam Abdoul-Anziz, Lukáš Jakabčin, Pierre Seppecher. Homogenization of an elastic material reinforced by very strong fibers arranged along a periodic lattice. Proceedings of the Royal Society A: Mathematical, Physical and Engineering Sciences, 2021, 10.1098/rspa.2020.0620 . hal-02482837

\section{HAL Id: hal-02482837 \\ https://hal-univ-tln.archives-ouvertes.fr/hal-02482837}

Submitted on 18 Feb 2020

HAL is a multi-disciplinary open access archive for the deposit and dissemination of scientific research documents, whether they are published or not. The documents may come from teaching and research institutions in France or abroad, or from public or private research centers.
L'archive ouverte pluridisciplinaire HAL, est destinée au dépôt et à la diffusion de documents scientifiques de niveau recherche, publiés ou non, émanant des établissements d'enseignement et de recherche français ou étrangers, des laboratoires publics ou privés. 


\title{
Homogenization of an elastic material reinforced by very strong fibers arranged along a periodic lattice.
}

\author{
Houssam Abdoul-Anziz ${ }^{1}$, Lukáš Jakabčin ${ }^{2,3}$ and Pierre Seppecher ${ }^{3}$
}

February 18, 2020

\section{Contents}

\begin{tabular}{llr}
\hline & Introduction & 2
\end{tabular}

2 Initial problem, description of the geometry 3

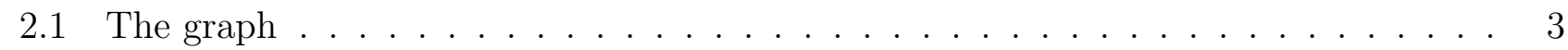

2.2 The $2 \mathrm{D}$ elastic problem . . . . . . . . . . . . . . . . . . . . . . 6

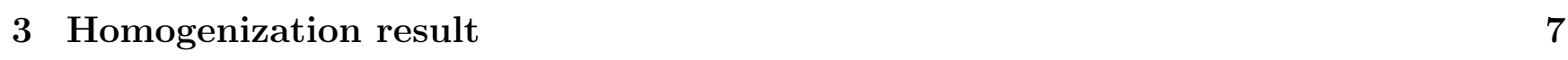

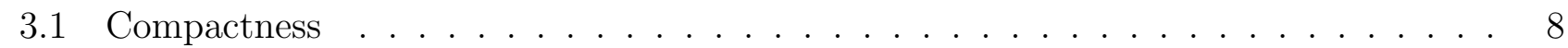

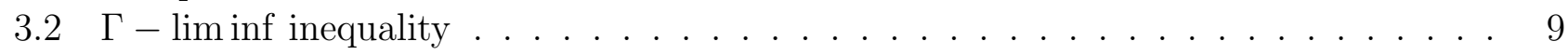

3.3 Construction of an approximating sequence . . . . . . . . . . . . . . . . . . . . . . 10

\begin{tabular}{|lr}
\hline Conclusion & 16
\end{tabular}

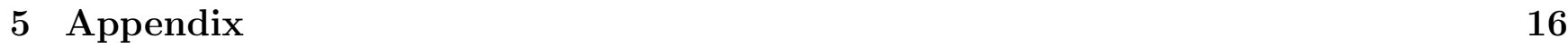

\begin{abstract}
We provide in this paper homogenization results for the $\mathrm{L}^{2}$-topology leading to complete strain-gradient models and generalized continua. Actually we extend to the $\mathrm{L}^{2}$-topology the results obtained in [1] using a topology adapted to minimization problems set in varying domains. Contrary to [1] we consider elastic lattices embedded in an soft elastic matrix. Thus our study is placed in the usual framework of homogenization. The contrast between the elastic stiffnesses of the matrix and the reinforcement zone is assumed to be very large. We prove that a suitable choice of the stiffness on the weak part ensures the compactness of minimizing sequences while the energy contained in the matrix disappears at the limit: the $\Gamma$-limit energies we obtain are identical to those obtained in [1].
\end{abstract}

\footnotetext{
${ }^{1}$ Laboratoire Modélisation et Simulation Multi-Echelle (MSME), UMR 8208 CNRS, Université Gustave Eiffel. Email habdoulanziz@yahoo.fr

${ }^{2}$ Institut de Mathématiques de Marseille, Université Aix-Marseille, Technopôle Château-Gombert 39, rue F. Joliot Curie 13453 Marseille Cedex 13, lukas.jakabcin@univ-amu.fr

${ }^{3}$ Institut de Mathématiques de Toulon, Université de Toulon, BP 20132, 83957 La Garde Cedex, France. Email: seppecher@imath.fr
} 


\section{Introduction}

Homogenization theory of elastic periodic materials has generated a huge literature in the last decades (see for instance [23, 9, 6]). A part of it focuses on a class of the elastic periodic materials where the contrast of the elastic parameters of the material is so large that is can become comparable with the ratio of microscopic and macroscopic lengths. In this high-contrast case, the assumption of separation of scales must be revisited and the effective material which can result from the homogenization process may depart from standard elasticity theory.

First examples of such homogenization results were given in [22] in the three-dimensional case and in [14] in the two-dimensional case. In these papers a material periodically reinforced with parallel, very thin and very stiff fibers was studied. Other examples based on pantographic structures were given in [5, 4, 12, 18, 24].

When such highly contrasted structures are considered, the effective mechanical behavior may be not of the classical Cauchy elasticity type as it may contain non-local effects [10, 11, 1] or higher-order gradient effects [22, 14, 1]. The whole class of effective behaviors which can be obtained through an asymptotic process has been completely characterized in [15, 16].

Periodic homogenization consists in introducing the ratio $\varepsilon$ of the "microscopic" periodic size (wavelength of the varying stiffness coefficients) to the "macroscopic" size of the domain. In standard periodic homogenization, it is assumed that any stiffness coefficient depends on $\varepsilon$ only through the rescaled space variable $\varepsilon^{-1} x$. In other words, the stiffness is fixed in the rescaled cell. Taking into account high contrast needs to allow the amplitude of the variations of the stiffness coefficients also to depend on $\varepsilon$. We emphasize that letting the space dependence of the coefficients in the rescaled cell still involve $\varepsilon$ is also important for getting interesting effective energies.

In [1, 2, 3], a large family of examples based on periodic lattices has been described. In these recent papers, in the framework of linear elasticity, a class of second-gradient models, including eventually extra variables has been obtained via $\Gamma$-convergence approach: the considered structures were made of a unique linear elastic material forming a periodic lattice. The space between the thin substructure was empty. The study of these structures was first reduced to the study of discrete problems related to the rigid displacements of the nodes of the lattice. Homogenization of the discrete problems then became a pure algebraic computation which led to strain-gradient models and generalized continua that is to models enriched with extra kinematic variables. These results were the first to give explicit examples of rigorous homogenization results in the whole set of strain-gradient models or generalized continua. However they are difficult to compare to other homogenization results as they use a different topology. Indeed the presence of voids requires that the external forces are concentrated on the lattice only. In [1, 2, 3] it was assumed that the external forces were applied in the vicinity of the nodes. The limit model is set in a limit domain $\Omega$. It is clear that the $\Gamma$-convergence theorem cannot be stated for the $\mathrm{L}^{2}(\Omega)$ topology like it is done in [15, 16, 22, 14.

Moreover, a higher-order homogenization procedure at the order $\varepsilon^{2}$ with second-gradient effects was introduced in [25, 7] revisiting the work of [8]. This procedure also needs that the material is nowhere degenerate and thus forbids the presence of voids. This procedure seems to be very robust: even when applied out of its validity domain, it still seem to give the right high-order stiffness tensors (see [19] for pantographs). In [21], it was numerically shown that this procedure applied to the elastic periodic structures considered in [1] with the addition of a compliant material inside the voids leads to the same limit second-gradient models as those obtained in [1]. So the question was open of the suitable choice of the stiffness of the compliant embedding matrix for these numerical results to be consolidated. 
The aim of the present paper is to answer this question by extending rigorously the results of [1] to a continuum elastic material without voids. In that case, the elastic problems are all set in a fixed domain $\Omega$ and external forces can be applied on the whole domain. We show that adding the compliant matrix has no consequence on the limit model obtained in [1]: for a suitable choice of the stiffness in the weak part, the energy concentrated in the compliant part disappears when passing to the $\Gamma$-limit.

The paper is organized as follows. We first describe, in Section 2, the geometry and the considered elasticity problem. We then state the homogenization result (Theorem 1 in in Section 3. For sake of clarity, the proof of this theorem is divided in three subsections. The compactness needs a careful estimation of the capacity of the lattice with respect to the whole domain. The $\Gamma$-lim inf inequality results easily from the results of [1] as it is clear that the energy considered in this paper is larger than the energy considered there. The $\Gamma$-lim sup inequality is obtained by the explicit construction of an approximating sequence. The novelty lies essentially in the need of a Lipschitz extension of a displacement field initially defined is the reinforcement lattice only and to the introduction of a triangulated augmented lattice which makes the construction easier.

\section{Initial problem, description of the geometry}

\section{$2.1 \quad$ The graph}

In the two-dimensional space endowed with an orthonormal coordinate system $\left(0, e^{1}, e^{2}\right)$, we consider a weak material periodically reinforced by very strong thin fibers. The fibers form a periodic planar graph that we first describe. We follow closely the description of [1] or [20]. A prototype cell $\mathrm{Y}=\left(-\frac{1}{2}, \frac{1}{2}\right)^{2}$ contains a finite number $K$ of nodes the position of which is denoted $y_{r}$, $r \in\{1, \ldots, K\}$. Without loss of generality we assum $1^{1}$ that $\sum_{r \in\{1, \ldots, K\}} y_{r}=0$. This cell is scaled by a small parameter $\varepsilon$ (assumed to be of the form $\varepsilon=\frac{1}{2 N^{\varepsilon}+1}$ with $N^{\varepsilon} \in \mathbb{N}$ ) and reproduced in order to make a tiling of the domain $\Omega=\left(-\frac{1}{2}, \frac{1}{2}\right)^{2}$. The nodes of the graph are thus the points $y_{I, s}^{\varepsilon}:=\varepsilon\left(y_{s}+i e^{1}+j e^{2}\right)$ for $I=(i, j) \in \mathscr{I}^{\varepsilon}:=\left\{-N^{\varepsilon}, \ldots, N^{\varepsilon}\right\}^{2}$ and the cells are the squares

$$
C_{I}^{\varepsilon}:=\varepsilon\left(i e^{1}+j e^{2}+\mathrm{Y}\right)
$$

The reinforcing fibers (called edges in the sequel) may connect some node $r$ of a cell $I$ with some other node $s$ of the same cell or of one of its closest neighbor ${ }^{2} I+p$ with $p \in \mathcal{P}:=\{-1,0,1\}^{2}$. For any $p=\left(p_{1}, p_{2}\right)$ in $\mathcal{P}$ we denote $\mathbf{p}:=p_{1} e^{1}+p_{2} e^{2}$ the corresponding vector so that $y_{I+p, r}^{\varepsilon}=y_{I, r}^{\varepsilon}+\varepsilon \mathbf{p}$.

The set of edges is characterized by a subset $\mathscr{A} \subset \mathcal{P} \times\{1, \ldots, K\}^{2}$ : node $y_{I, r}^{\varepsilon}$ is connected to node $y_{I+p, s}^{\varepsilon}$ if and only if $(p, r, s) \in \mathscr{A}$. The considered graph $G^{\varepsilon}$ is the union over all $I$ in $\mathscr{I}^{\varepsilon}$ and all $(p, r, s)$ in $\mathscr{A}$ of the segments $\left[y_{I, r}^{\varepsilon}, y_{I+p, s}^{\varepsilon}\right]$. The set $\mathscr{A}$ is chosen in such a way that (i) there is no crossing or overlapping of edges corresponding to different elements of $\mathscr{A}$, (ii) the resulting graph is connected.

The complementary of the graph is a union of polygons which take a finite number of shapes. By introducing a set $\tilde{\mathscr{A}}$ with $\mathscr{A} \subset \tilde{\mathscr{A}} \subset \mathcal{P} \times\{1, \ldots, K\}^{2}$, it is easy to add some edges in the initial graph in order to get a triangulated graph. This augmented graph, denoted $\tilde{G}^{\varepsilon}$, is introduced for purely technical reasons. The complementary of the augmented graph is a union of triangles

\footnotetext{
1 The symbol $\sum$ stands for the mean value (e.g. $\sum_{r \in\{1, \ldots, K\}} y_{r}=\frac{1}{K} \sum_{r \in\{1, \ldots, K\}} y_{r}$ ).

${ }^{2}$ It has been shown in [1] that restricting the interactions to the closest neighbors is not an actual restriction.
} 
which take a finite number of shapes. We call $\alpha_{\text {min }}>0$ the smallest angle appearing in all these triangles.

For any $(p, r, s)$ in $\mathscr{A}$ or $\tilde{\mathscr{A}}$ we introduce the rescaled length and direction of the edge by setting

$$
\ell_{p, r, s}:=\varepsilon^{-1}\left\|y_{I+p, s}^{\varepsilon}-y_{I, r}^{\varepsilon}\right\| \quad \text { and } \quad \tau_{p, r, s}:=\frac{y_{I+p, s}^{\varepsilon}-y_{I, r}^{\varepsilon}}{\varepsilon \ell_{p, r, s}}
$$

We also introduce $\ell_{\text {min }}:=\min _{(p, r, s) \in \mathscr{A}} \ell_{p, r, s}$ and $\ell_{\text {max }}:=\max _{(p, r, s) \in \mathscr{A}} \ell_{p, r, s}$.

The fibers are assumed to have width $\beta \varepsilon^{2}$ with $\beta>0$. Thus we introduce

$$
\Omega^{\varepsilon}:=\left\{x \in \Omega ; d\left(x, G^{\varepsilon}\right)<\frac{\beta}{2} \varepsilon^{2}\right\}, \quad \widetilde{\Omega}^{\varepsilon}:=\left\{x \in \Omega ; d\left(x, \tilde{G}^{\varepsilon}\right)<\frac{\beta}{2} \varepsilon^{2}\right\}
$$

and the reinforcement domain is the "thickened graph" $\Omega^{\varepsilon}$.

Let us describe some parts (see Fig. 2) of $\widetilde{\Omega}^{\varepsilon}$ which will play an essential role in what follows. First, for any $I \in \mathscr{I}^{\varepsilon}$ and $s \in\{1, \ldots, K\}$, we introduce the balls

$$
B_{I, s}^{\varepsilon}:=\left\{x \in \Omega ; d\left(x, y_{I, s}^{\varepsilon}\right)<\frac{\beta}{2} \varepsilon^{2}\right\}
$$

which will approximately act like small rigid bodies. Then, for any $I \in \mathscr{I}^{\varepsilon}$ and $(p, r, s) \in \tilde{\mathscr{A}}$, we introduce the rectangles ${ }^{3}$

$$
R_{I, p, r, s}^{\varepsilon}:=\left\{\frac{y_{I, r}^{\varepsilon}+y_{I+p, s}^{\varepsilon}}{2}+x_{1} \tau_{p, r, s}+x_{2} \tau_{p, r, s}^{\perp}:\left(x_{1}, x_{2}\right) \in\left[-\frac{\ell_{p, r, s}}{2} \varepsilon, \frac{\ell_{p, r, s}}{2} \varepsilon\right] \times\left[-\frac{\beta}{2} \varepsilon^{2}, \frac{\beta}{2} \varepsilon^{2}\right]\right\} .
$$

in which a Bernoulli-Navier displacement will be a good approximation of the actual displacement field. These rectangles overlap in the vicinity of any common node. It is easy to check that such an overlapping is excluded in the part of the rectangle where $x_{1}$ is restricted to the interval

$$
x_{1} \in\left[-\frac{\gamma^{\varepsilon} \ell_{p, r, s}}{2} \varepsilon, \frac{\gamma^{\varepsilon} \ell_{p, r, s}}{2} \varepsilon\right] \quad \text { with } \quad \gamma^{\varepsilon}:=1-\frac{\beta}{\ell_{\min } \tan \left(\alpha_{\min } / 2\right)} \varepsilon .
$$

The meaning of parameter $\gamma^{\varepsilon}$ is illustrated in Fig. 2 .

Example 1. Let us illustrate the description of the geometry on a specific example. This example has been first studied in [21] where it has been proved to lead to a non-local limit energy of Cosserat type. The material is reinforced by a thin square grid braced by diagonals in one square over four (see Fig. 1 and Fig. 2).

One can describe this situation by considering a cell containing four $(K=4)$ nodes: $y_{1}=$ $\left(-\frac{1}{4},-\frac{1}{4}\right) y_{2}=\left(\frac{1}{4},-\frac{1}{4}\right), y_{3}=\left(\frac{1}{4}, \frac{1}{4}\right), y_{4}=\left(-\frac{1}{4}, \frac{1}{4}\right)$. The reinforcement structures consist in all the rectangles joining nodes $y_{1}$ and $y_{2}, y_{1}$ and $y_{3}, y_{1}$ and $y_{4}, y_{2}$ and $y_{3}, y_{3}$ and $y_{4}$ inside the cell, the rectangles joining nodes $y_{2}$ and $y_{3}$ of the cell with respectively nodes $y_{1}$ and $y_{4}$ of the next cell following $e^{1}$, and finally the rectangles joining nodes $y_{3}$ and $y_{4}$ of the cell with respectively nodes $y_{2}$ and $y_{1}$ of the next cell following $e^{2}$. All this is resumed by setting:

$\mathscr{A}=\{(0,1,2),(0,1,3),(0,1,4),(0,2,3),(0,3,4),((1,0), 2,1),((1,0), 3,4),((0,1), 3,2),((0,1), 4,1)\}$.

\footnotetext{
${ }^{3}$ For any vector $\tau$, notation $\tau^{\perp}$ stands for the vector obtained from $\tau$ by a rotation of angle $+\frac{\pi}{2}$.
} 


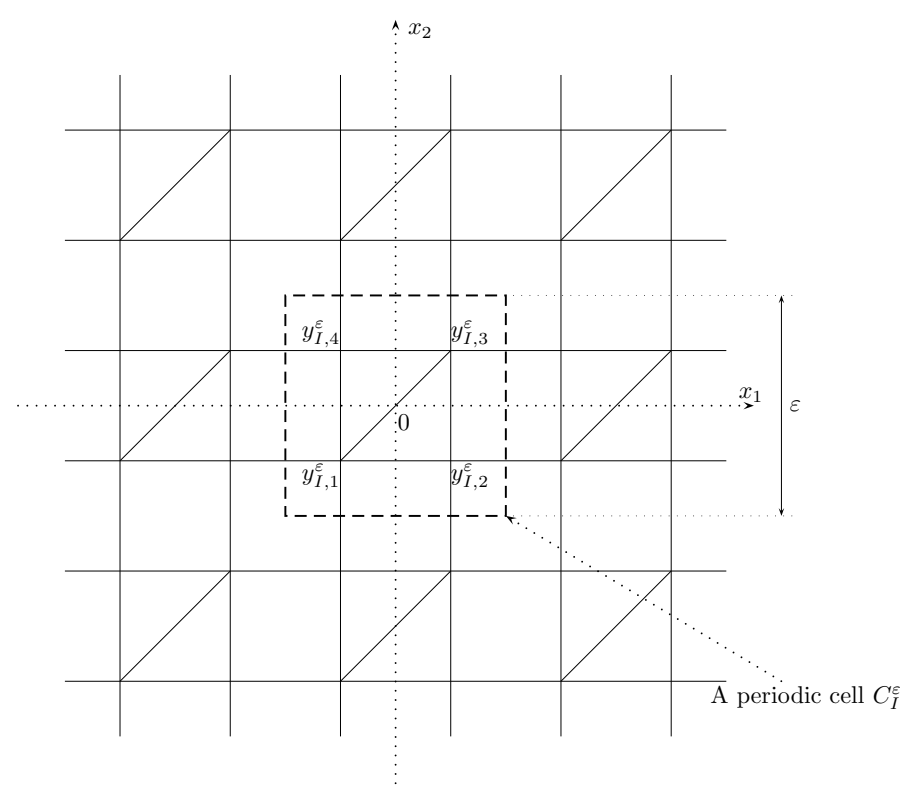

Figure 1: An example of periodic graph.



Figure 2: The rescaled prototype cell corresponding to Fig. 1. The reinforcement zone is darker. We illustrate in this figure the fact that the central part with length $\gamma^{\varepsilon} \ell_{p, r, s}$ of any connecting rectangle of length $\ell_{p, r, s}$ is not overlapping other reinforcement zone. 
In that case, the augmented (triangulated) graph could simply be obtained by adding three missing diagonals

$$
\tilde{\mathscr{A}}=\mathscr{A} \cup\{((1,0), 2,4),((0,1), 4,2),((1,1), 3,1)\} .
$$

In Fig. 2 is represented the rescaled cell. One must notice that, despite the rescaling, the geometry still depend on $\varepsilon$. Indeed the actual thickness of the reinforcement structures being $\beta \varepsilon^{2}$, the rescaled thickness is $\beta \varepsilon$. This is a rarely explored situation in periodic homogenization.

Let us make a last remark about the set $\widetilde{\Omega}^{\varepsilon}$. Let $x$ and $y$ be two points in $\widetilde{\Omega}^{\varepsilon}$ then there exists a piece-wise $C^{1}$ path in $\widetilde{\Omega}^{\varepsilon}$ connecting these two points whose length is smaller or equal to $\frac{\sqrt{2}\|y-x\|}{\sqrt{1-\cos \left(\alpha_{\min }\right)}}$. Indeed any part of the segment $[x, y]$ which lies in a triangle part of the complementary of $\widetilde{\Omega}^{\varepsilon}$ can be replaced by a path along the boundary of the triangle. It is easy to check that this operation does not multiply the length of the path by more than $\sqrt{\frac{2}{1-\cos \left(\alpha_{\min }\right)}}$. The consequence is that, whenever $u$ is a $C^{1}$ function on $\widetilde{\Omega}^{\varepsilon}$ satisfying $\|\nabla u\| \leq C$ then $u$ is a $\frac{\sqrt{2} C}{\sqrt{1-\cos \left(\alpha_{\min }\right)}}$-Lipschitz function.

\subsection{The $2 \mathrm{D}$ elastic problem}

We study the highly contrasted linear elastic problem 4 : $\inf _{u}\left\{\mathscr{E}_{\varepsilon}(u)\right\}$ where

$$
\mathscr{E}_{\varepsilon}(u):= \begin{cases}\int_{\Omega} Y(x)\left(\frac{1}{2(1+\nu)}\|e(u)\|^{2}+\frac{\nu}{2\left(1-\nu^{2}\right)}(\operatorname{tr}(e(u)))^{2}\right) d x & \text { if } u \in \mathrm{H}^{1}\left(\Omega, \mathbb{R}^{2}\right), \\ +\infty & \text { if } u \in \mathrm{L}^{2}\left(\Omega^{\varepsilon}, \mathbb{R}^{2}\right) \backslash \mathrm{H}^{1}\left(\Omega^{\varepsilon}, \mathbb{R}^{2}\right)\end{cases}
$$

and the Young modulus $Y$ takes a very large value inside $\Omega^{\varepsilon}$ and a very small one outside of it:

$$
Y(x)= \begin{cases}Y_{0} \varepsilon^{-3} & \text { if } x \in \Omega^{\varepsilon} \\ Y_{1} \varepsilon^{a} & \text { with } 0<a<2, \text { otherwise. }\end{cases}
$$

We study here the non degenerate case where $Y_{1}>0$ and $Y_{0}>0$ and the Poisson coefficient $\nu$, assumed to be constant for sake of simplicity, satisfies $-1<\nu<1$ so that

$$
C\|e(u)\|^{2} \leq \frac{1}{2(1+\nu)}\|e(u)\|^{2}+\frac{\nu}{2\left(1-\nu^{2}\right)}(\operatorname{tr}(e(u)))^{2} \leq \frac{1}{4}\|e(u)\|^{2}
$$

with

$$
C=\frac{1}{2 \min (1+\nu, 1-\nu)}>0
$$

Let us denote $\mathscr{E}_{\varepsilon}^{0}$ the functional defined like $(6)$ but with $Y_{1}=0$. In other words

$$
\mathscr{E}_{\varepsilon}^{0}(u):= \begin{cases}\int_{\Omega^{\varepsilon}} Y(x)\left(\frac{1}{2(1+\nu)}\|e(u)\|^{2}+\frac{\nu}{2\left(1-\nu^{2}\right)}(\operatorname{tr}(e(u)))^{2}\right) d x & \text { if } u \in \mathrm{H}^{1}\left(\Omega^{\varepsilon}, \mathbb{R}^{2}\right), \\ +\infty & \text { if } u \in \mathrm{L}^{2}\left(\Omega^{\varepsilon}, \mathbb{R}^{2}\right) \backslash \mathrm{H}^{1}\left(\Omega^{\varepsilon}, \mathbb{R}^{2}\right) .\end{cases}
$$

The functional $\mathscr{E}_{\varepsilon}^{0}$ has been studied in [1] in a two-step process: it has been first proved that it shares the same asymptotic behavior than the following discrete functional $E_{\varepsilon}+F_{\varepsilon}$ acting on

\footnotetext{
${ }^{4}$ Here $e(u)$ denotes the symmetric part of the gradient of $u\left(e(u)=\left(\nabla u+(\nabla u)^{t}\right) / 2\right.$ is the linearized strain tensor) and $\operatorname{tr}(e(u))$ denotes the trace of the matrix $e(u)$.
} 
families $\left(U_{I, r}, \theta_{I, r}\right)$ of rigid motions defined at the nodes of the graph $\left(U_{I, r}\right.$ being vector valued while $\theta_{I, r}$ is scalar):

$$
\begin{aligned}
& E_{\varepsilon}(U):=\sum_{(I, p, r, s) \in \mathcal{I}^{\varepsilon} \times \mathcal{A}} \frac{Y_{0} \beta}{\ell_{p, r, s}}\left(\frac{U_{I+p, s}-U_{I, r}}{\varepsilon} \cdot \tau_{p, r, s}\right)^{2}, \\
& F_{\varepsilon}(U, \theta):=\varepsilon^{2} \sum_{(I, p, r, s) \in \mathcal{I}^{\varepsilon} \times \mathcal{A}} \frac{Y_{0} \beta^{3}}{3 \ell_{p, r, s}}\left(3\left(\theta_{I+p, s}+\theta_{I, r}-\frac{2}{\ell_{p, r, s}} \frac{U_{I+p, s}-U_{I, r}}{\varepsilon} \cdot \tau_{p, r, s}^{\perp}\right)^{2}+\left(\theta_{I+p, s}-\theta_{I, r}\right)^{2}\right) .
\end{aligned}
$$

Then it has been proved that, for a suitable notion of convergence, $E_{\varepsilon}+F_{\varepsilon}$ converges to

$$
\mathscr{E}(u):=\inf _{w, v, \theta}\left\{\bar{E}\left(w, \xi_{u, v}\right)+\bar{F}\left(v, \eta_{u}, \theta\right) ; \bar{E}\left(v, \eta_{u}\right)=0\right\} .
$$

where $\bar{E}$ and $\bar{F}$ are the continuous counterparts of $E_{\varepsilon}$ and $F_{\varepsilon}$, namely

$$
\begin{aligned}
\bar{E}(v, \eta):=\int_{\Omega} \sum_{(p, r, s)} \frac{Y_{0} \beta}{\ell_{p, r, s}}\left(\left(v_{s}(x)-v_{r}(x)+\eta_{p, s}(x)\right) \cdot \tau_{p, r, s}\right)^{2} d x \\
\bar{F}(v, \eta, \theta):=\int_{\Omega} \sum_{(p, r, s)} \frac{Y_{0} \beta^{3}}{3 \ell_{p, r, s}}\left(3 \left(\theta_{s}(x)+\theta_{r}(x)\right.\right. \\
\left.\left.\quad-\frac{2}{\ell_{p, r, s}}\left(v_{s}(x)-v_{r}(x)+\eta_{p, s}(x)\right) \cdot \tau_{p, r, s}^{\perp}\right)^{2}+\left(\theta_{s}(x)-\theta_{r}(x)\right)^{2}\right)
\end{aligned}
$$

and $\eta_{u}$ and $\xi_{u, v}$ stand for the families defined by

$$
\begin{aligned}
& \left(\eta_{u}\right)_{p, s}:=\nabla u \cdot \mathbf{p}, \\
& \left(\xi_{u, v}\right)_{p, s}=\nabla v_{s} \cdot \mathbf{p}+\frac{1}{2} \nabla \nabla u \cdot \mathbf{p} \cdot \mathbf{p} .
\end{aligned}
$$

Note that the functionals in (11) are defined for any $u, v, w, \theta$ in $\mathrm{L}^{2}(\Omega)$ and, by convention, take value $+\infty$ whenever the integrals are divergent.

\section{Homogenization result}

The reader may refer to [17, 13] for the properties of $\Gamma$-convergence.

Theorem 1. The sequence $\mathscr{E}_{\varepsilon}$ defined by (6) $\Gamma$-converges to $\mathscr{E}$ defined by (11) for the weak- $\mathrm{L}^{2}(\Omega)$ topology:

(i) Any sequence $u^{\varepsilon}$ with zero mean rigid motion $\left(\int_{\Omega} u^{\varepsilon}(x) d x=0\right.$ and $\left.\int_{\Omega} x \times u^{\varepsilon}(x) d x=0\right)$ and with bounded energy $\left(\mathscr{E}_{\varepsilon}\left(u^{\varepsilon}\right) \leq M\right)$ is relatively compact in $\mathrm{L}^{2}(\Omega)$.

(ii) For any sequence $u^{\varepsilon}$ converging weakly in $\mathrm{L}^{2}(\Omega)$ to some function $u$, we have $\lim \inf \mathscr{E}_{\varepsilon}\left(u^{\varepsilon}\right) \geq$ $\mathscr{E}(u)$.

(iii) For any $u$ such that $\mathscr{E}(u)<+\infty$, there exists a sequence $u^{\varepsilon}$ converging to $u$ in $\mathrm{L}^{2}(\Omega)$ and such that $\lim \sup \mathscr{E}_{\varepsilon}\left(u^{\varepsilon}\right) \leq \mathscr{E}(u)$.

The energy we consider here is clearly larger than the energy $\mathscr{E}_{\varepsilon} 0$ considered in [1]. The compactness property established there for some weak convergence of measures will help us to prove compactness in $L^{2}(\Omega)$ of sequences with bounded energy. Moreover, as we expect the same $\Gamma$-limit for both cases, our $\Gamma$-lim inf inequality will essentially be a consequence of the $\Gamma$-lim inf inequality established in [1]. For sake of clarity, the three assertions of Theorem 1 are proved in the following three subsections. 


\subsection{Compactness}

We will use the following lemma whose proof, essentially based on the estimation of the capacity of the periodic network of balls $\left(B_{I, r}^{\varepsilon}\right)_{I \in \mathscr{I}^{\varepsilon}}$, is postponed to the Appendix.

Lemma 1. To any $u \in \mathrm{H}^{1}(\Omega)$, let us associate the quantities $\bar{u}_{I, s}^{\varepsilon}:=f_{B_{I, s}^{\varepsilon}} u(x) d x$ and the piecewise constant function

$$
\tilde{u}_{s}^{\varepsilon}(x):=\sum_{I \in \mathscr{I}^{\varepsilon}} \bar{u}_{I, s}^{\varepsilon} 1_{C_{I}^{\varepsilon}}(x)
$$

There exists a constant $C$ such that, for any $u \in \mathrm{H}^{1}(\Omega)$,

$$
\left\|u-\tilde{u}_{s}^{\varepsilon}\right\|_{L^{2}(\Omega)}^{2} \leq C \varepsilon^{2}|\ln (\varepsilon)|\|\nabla u\|_{L^{2}(\Omega)}^{2} .
$$

The compactness result can now be established.

Proof. Let $u^{\varepsilon}$ satisfying

$$
\int_{\Omega} u^{\varepsilon}(x) d x=0, \quad \int_{\Omega} x \times u^{\varepsilon}(x) d x=0 \quad \text { and } \quad \mathscr{E}_{\varepsilon}\left(u^{\varepsilon}\right) \leq M .
$$

Using (7) and $Y(x) \geq Y_{1} \varepsilon^{a}$, last inequality implies $Y_{1} \varepsilon^{a} C \int_{\Omega}\left\|e\left(u^{\varepsilon}\right)(x)\right\|^{2} d x \leq M$. As $u^{\varepsilon}$ has zero mean rigid motion, Korn inequality implies

$$
\int_{\Omega}\left\|\nabla u^{\varepsilon}(x)\right\|^{2} d x \leq K \int_{\Omega}\left\|e\left(u^{\varepsilon}\right)(x)\right\|^{2} d x \leq \frac{K M}{C Y_{1} \varepsilon^{a}} .
$$

Let us consider the family of vectors and the piece-wise constant functions

$$
\bar{u}_{I, s}^{\varepsilon}:=\int_{B_{I, s}^{\varepsilon}} u^{\varepsilon}(x) d x \quad \text { and } \quad \tilde{u}_{s}^{\varepsilon}(x):=\sum_{I \in \mathscr{I}^{\varepsilon}} \bar{u}_{I, s}^{\varepsilon} 1_{C_{I}^{\varepsilon}}(x) .
$$

Lemma 1 states that $\left\|u^{\varepsilon}-\tilde{u}_{s}^{\varepsilon}\right\|_{L^{2}(\Omega)}^{2} \leq C \varepsilon^{2}|\ln (\varepsilon)|\left\|\nabla u^{\varepsilon}\right\|_{L^{2}(\Omega)}^{2}$ and thus

$$
\left\|u^{\varepsilon}-\tilde{u}_{s}^{\varepsilon}\right\|_{L^{2}(\Omega)}^{2} \leq C \varepsilon^{2-a}|\ln (\varepsilon)| \rightarrow 0 .
$$

Clearly, omitting the elastic energy concentrated in the weak part of the material, we have $\mathscr{E}_{\varepsilon}\left(u^{\varepsilon}\right) \geq \mathscr{E}_{\varepsilon}^{0}$. It is proved in Theorem 1 of [1] that, for any $\eta>0$ and for $\varepsilon$ small enough,

$$
\mathscr{E}_{\varepsilon}^{0}\left(u^{\varepsilon}\right) \geq E_{\varepsilon}\left(\left(\bar{u}_{I, s}^{\varepsilon}\right)\right)+F_{\varepsilon}\left(\left(\bar{u}_{I, s}^{\varepsilon}\right),\left(\phi_{I, s}^{\varepsilon}\right)\right)-\eta
$$

where $\phi_{I, s}^{\varepsilon}:=\int_{B_{I, s}^{\varepsilon}} \frac{\partial_{1} u_{2}^{\varepsilon}-\partial_{2} u_{1}^{\varepsilon}}{2}(x) d x$. Inequality 20 is invariant when subtracting a rigid motion. We set

$a^{\varepsilon}:=\sum_{I \in \mathscr{I} \varepsilon} \sum_{s=1}^{K} \phi_{I, s}^{\varepsilon}, \quad b^{\varepsilon}:=\sum_{I \in \mathscr{I} \varepsilon} \sum_{s=1}^{K} \bar{u}_{I, s}^{\varepsilon}, \quad \theta_{I, s}^{\varepsilon}=\phi_{I, s}^{\varepsilon}-a^{\varepsilon}, \quad r^{\varepsilon}(x):=a^{\varepsilon} x^{\perp}+b^{\varepsilon} \quad$ and $\quad v^{\varepsilon}=u^{\varepsilon}-r^{\varepsilon}$,

so that the families $\left(\bar{v}_{I, s}^{\varepsilon}\right)$ and $\left(\theta_{I, s}^{\varepsilon}\right)$ satisfy $\sum_{I \in \mathscr{I} \varepsilon} \sum_{s=1}^{K} \bar{v}_{I, s}^{\varepsilon}=0$ and $\sum_{I \in \mathscr{I} \varepsilon} \sum_{s=1}^{K} \theta_{I, s}^{\varepsilon}=0$ and we still have $E_{\varepsilon}\left(\left(\bar{v}_{I, s}^{\varepsilon}\right)\right)+F_{\varepsilon}\left(\left(\bar{v}_{I, s}^{\varepsilon}\right),\left(\theta_{I, s}^{\varepsilon}\right)\right) \leq M+\eta$. Here we associate to $v^{\varepsilon}$ and $r^{\varepsilon}$ the quantities $\bar{v}_{I, s}^{\varepsilon}$ 
and $\bar{r}_{I, s}^{\varepsilon}$ and the functions $\tilde{v}^{\varepsilon}$ and $\tilde{r}^{\varepsilon}$ like in (18). Lemma 4 of [1] states that, for any $s, \sum_{I}\left\|\bar{v}_{I, s}^{\varepsilon}\right\|^{2}$ is uniformly bounded. Thus $\left\|\tilde{u}_{s}^{\varepsilon}-\tilde{r}_{s}^{\varepsilon}\right\|_{\mathrm{L}^{2}(\Omega)}^{2}=\left\|\tilde{v}_{s}^{\varepsilon}\right\|_{\mathrm{L}^{2}(\Omega)}^{2} \leq C$. Using (19) we get by triangle inequality $\left\|u^{\varepsilon}-\tilde{r}_{s}^{\varepsilon}\right\|_{\mathrm{L}^{2}(\Omega)} \leq C$. As $f_{\Omega} u^{\varepsilon}(x) d x=0$,

$$
b^{\varepsilon}=\sum_{I \in \mathscr{I}^{\varepsilon}} \sum_{s=1}^{K} r^{\varepsilon}\left(y_{I, s}^{\varepsilon}\right)=\sum_{I \in \mathscr{I}^{\varepsilon}} \sum_{s=1}^{K} \bar{r}_{I, s}^{\varepsilon}=\sum_{s=1}^{K} f_{\Omega} \tilde{r}_{s}^{\varepsilon}(x) d x=\sum_{s=1}^{K} f_{\Omega}\left(u^{\varepsilon}(x)-\tilde{r}_{s}^{\varepsilon}(x)\right) d x
$$

and we first deduce $\left\|b^{\varepsilon}\right\| \leq C$. As $f_{\Omega} x d x=0$ we have $\int_{\Omega} x \times b^{\varepsilon} d x=0$ and, as we have assumed $f_{\Omega} x \times u^{\varepsilon} d x=0$, we can also deduce

$$
\left|a^{\varepsilon}\right| f_{\Omega}\|x\|^{2} d x=\left\|f_{\Omega} x \times\left(\tilde{r}_{s}^{\varepsilon}(x)-b^{\varepsilon}\right) d x\right\|=\left\|f_{\Omega} x \times\left(\tilde{r}_{s}^{\varepsilon}(x)-u^{\varepsilon}\right) d x\right\| \leq C .
$$

Hence $\left|a^{\varepsilon}\right|<C$. Therefore the sequence of rigid motions $r^{\varepsilon}$ is bounded in $\mathrm{L}^{2}(\Omega)$. On the other hand, we have

$$
\begin{aligned}
\left\|r^{\varepsilon}-\sum_{s} \tilde{r}_{s}^{\varepsilon}\right\|_{L^{2}(\Omega)}^{2} & =\sum_{s} \sum_{I} \underset{C_{I}^{\varepsilon}}{f}\left\|r^{\varepsilon}(x)-\bar{r}_{I, s}^{\varepsilon}\right\|^{2} d x \\
& =\sum_{s} \sum_{I}{\underset{C}{I}}_{f}\left\|a^{\varepsilon} x^{\perp}+b^{\varepsilon}-\left(a^{\varepsilon}\left(y_{I, s}^{\varepsilon}\right)^{\perp}+b^{\varepsilon}\right)\right\|^{2} d x \\
& =\left(a^{\varepsilon}\right)^{2} \sum_{s} \sum_{I} \underset{C_{I}^{\varepsilon}}{f}\left\|x-y_{I, s}^{\varepsilon}\right\|^{2} d x \leq \frac{\left(a^{\varepsilon}\right)^{2} \varepsilon^{2}}{2} .
\end{aligned}
$$

So, by triangle inequality, $u^{\varepsilon}$ is bounded in $\mathrm{L}^{2}(\Omega)$. Point(i) is proved.

\section{2 $\Gamma-\lim$ inf inequality}

Proof. Let now $u^{\varepsilon}$ be a sequence with bounded energy and converging weakly in $\mathrm{L}^{2}(\Omega)$ to some $u$. From estimation (19), we know that, for any $s \in\{1, \ldots, K\}, \tilde{u}_{s}^{\varepsilon}$ converges to $u$. For any $\varphi \in C^{0}\left(\mathbb{R}^{2}\right)$, we have

$$
\sum_{I} \bar{u}_{I, s}^{\varepsilon} \int_{C_{I}^{\varepsilon}} \varphi(x) d x=\int_{\Omega} \tilde{u}_{s}^{\varepsilon}(x) \varphi(x) d x \rightarrow \int_{\Omega} u(x) \varphi(x) d x .
$$

On the other hand $\int_{\Omega} \bar{u}_{I, s}^{\varepsilon}(x) \varphi(x) d x=\sum_{I} \bar{u}_{I, s}^{\varepsilon} \int_{C_{I}^{\varepsilon}} \varphi(x) d x$. As $\varphi$ is uniformly continuous on the compact $\bar{\Omega}$, there exists $C$ such that, for all $I, s$ and $x \in C_{I}^{\varepsilon},\left\|\varphi(x)-\varphi\left(y_{I, s}^{\varepsilon}\right)\right\| \leq C \varepsilon$. Hence

$$
\sum_{I} \bar{u}_{I, s}^{\varepsilon}\left|C_{I}^{\varepsilon}\right| \varphi\left(y_{I, s}^{\varepsilon}\right) \rightarrow \int_{\Omega} u(x) \varphi(x) d x
$$

In other words, we have the convergence in the sense of measures of the sequence of discrete measures

$$
\sum_{I} \bar{u}_{I, s}^{\varepsilon} \delta_{y_{I, s}^{\varepsilon}} \stackrel{*}{\rightarrow} u
$$


and we can apply the $\Gamma$-convergence Theorems 1 and 2 established in [1] which state respectively that

$$
\begin{aligned}
& \liminf _{\varepsilon \rightarrow 0} \mathscr{E}_{\varepsilon}^{0}\left(u^{\varepsilon}\right) \geq \inf _{\left(\theta_{I, s}^{\varepsilon}\right)} \liminf _{\varepsilon \rightarrow 0}\left(E_{\varepsilon}\left(\left(\bar{u}_{I, s}^{\varepsilon}\right)\right)+F_{\varepsilon}\left(\left(\bar{u}_{I, s}^{\varepsilon}\right),\left(\phi_{I, s}^{\varepsilon}\right)\right)\right), \\
& \liminf _{\varepsilon \rightarrow 0}\left(E_{\varepsilon}\left(\left(\bar{u}_{I, s}^{\varepsilon}\right)\right)+F_{\varepsilon}\left(\left(\bar{u}_{I, s}^{\varepsilon}\right),\left(\phi_{I, s}^{\varepsilon}\right)\right)\right) \geq \mathscr{E}(u) .
\end{aligned}
$$

Hence we get $\liminf _{\varepsilon \rightarrow 0} \mathscr{E}_{\varepsilon}\left(u^{\varepsilon}\right) \geq \liminf _{\varepsilon \rightarrow 0} \mathscr{E}_{\varepsilon}^{0}\left(u^{\varepsilon}\right) \geq \mathscr{E}(u)$ and point (ii) is proven.

\subsection{Construction of an approximating sequence}

Proof. In order to prove assertion (iii), we consider a function $u$ which satisfies $\mathcal{E}(u)<+\infty$ and, by a density argument, belongs to $C^{\infty}(\Omega)$. We follow the construction given in [1]. We just have to extend the fields defined there in the weak part of the material and to check that the energy in this zone is negligible. Thus we introduce $\left(v_{s}, w_{s}, \theta_{s}\right)$ such that $\mathcal{E}(u)=\bar{E}\left(w, \xi_{u, v}\right)+\bar{F}\left(v, \eta_{u}, \theta\right)$ and $\bar{E}\left(v, \eta_{u}\right)=0$. The coercivity and the lower semi-continuity of these functionals ensure the existence of these fields in $C^{\infty}(\Omega)$. We then define $U^{\varepsilon}$ and $\theta^{\varepsilon}$ by setting

$$
U_{I, s}^{\varepsilon}:=u\left(y_{I}^{\varepsilon}\right)+\varepsilon v_{s}\left(y_{I}^{\varepsilon}\right)+\varepsilon^{2} w_{s}\left(y_{I}^{\varepsilon}\right) \quad \text { and } \quad \theta_{I, s}^{\varepsilon}:=\theta_{s}\left(y_{I}^{\varepsilon}\right)
$$

Let $M \in \mathbb{R}$ which, for any $s \in\{1 \ldots K\}$, bounds uniformly the norms

$$
\|u\|, \quad\|\nabla u\|, \quad\|\nabla \nabla u\|, \quad\|\nabla \nabla \nabla u\|, \quad\left\|v_{s}\right\|, \quad\left\|\nabla v_{s}\right\|, \quad\left\|\nabla \nabla v_{s}\right\|, \quad\left\|w_{s}\right\| \quad \text { and } \quad\left\|\nabla w_{s}\right\| .
$$

We have, for any $(p, r, s) \in \tilde{\mathscr{A}}$,

$$
\left\|\frac{U_{I+p, s}^{\varepsilon}-U_{I, r}^{\varepsilon}}{\varepsilon}\right\| \leq C M, \quad\left|\theta_{I, r}^{\varepsilon}\right| \leq M, \quad\left|\theta_{I+p, s}^{\varepsilon}\right| \leq M,
$$

where the constant $C$ depends only on $\ell_{\max }:=\max _{(p, r, s) \in \tilde{\mathscr{A}}}\left(\ell_{p, r, s}\right)$.

Finally we define the function $u^{\varepsilon}$ on $\Omega$ by parts

- On each ball $B_{I, r}^{\varepsilon}$ : we set $u^{\varepsilon}(x):=U_{I, r}^{\varepsilon}+\theta_{I, r}^{\varepsilon}\left(x-y_{I, r}^{\varepsilon}\right)^{\perp}$.

- On each rectangle $R_{I, p, r, s}^{\varepsilon}$ corresponding to an edge $(p, r, s)$ in $\tilde{A}$ : the construction of $u^{\varepsilon}$ is more cumbersome. In order to simplify the notation, we drop the indices $(I, p, r, s)$ by using a suitable orthonormal coordinate system $\left(O, \tilde{e}^{1}, \tilde{e}^{2}\right)$ with origin at the middle of the edge $O:=\left(y_{I, r}^{\varepsilon}+y_{I+p, s}^{\varepsilon}\right) / 2$ and with vector $\tilde{e}^{1}:=\frac{y_{I+p, s}^{\varepsilon}-y_{I, r}^{\varepsilon}}{\left\|y_{I+p, s}^{\varepsilon}-y_{I, r}^{\varepsilon}\right\|}=\tau_{p, r, s}$ along the direction of the edge. Using this coordinate system the rectangle $R_{I, p, r, s}^{\varepsilon}$ reads

$$
R_{I, p, r, s}^{\varepsilon}=\left\{x=\varepsilon x_{1} \tilde{e}^{1}+\varepsilon^{2} x_{2} \tilde{e}^{2}:\left(x_{1}, x_{2}\right) \in\left(-\frac{\ell_{p, r s}}{2}, \frac{\ell_{p, r s}}{2}\right) \times\left(-\frac{\beta}{2}, \frac{\beta}{2}\right)\right\} .
$$

For simplifying further the notation we also set

$$
\theta_{m}:=\varepsilon^{-1} \frac{\left(U_{I+p, s}^{\varepsilon}-U_{I, r}^{\varepsilon}\right) \cdot \tilde{e}^{2}}{\gamma^{\varepsilon} \ell_{p, r, s}}-\frac{\left(1-\gamma^{\varepsilon}\right)\left(\theta_{I+p, s}^{\varepsilon}+\theta_{I, r}^{\varepsilon}\right)}{2 \gamma^{\varepsilon}},
$$




$$
U_{m}:=\frac{U_{I+p, s}^{\varepsilon}+U_{I, r}^{\varepsilon}}{2}-\varepsilon \frac{\left(1-\gamma^{\varepsilon}\right) \ell_{p, r, s}\left(\theta_{I+p, s}^{\varepsilon}-\theta_{I, r}^{\varepsilon}\right)}{4} \tilde{e}^{2}
$$

and $U^{-}:=U_{I, r}^{\varepsilon}-U_{m}, U^{+}:=U_{I+p, s}^{\varepsilon}-U_{m}, \theta^{-}:=\theta_{I, r}^{\varepsilon}-\theta_{m}, \theta^{+}:=\theta_{I+p, s}^{\varepsilon}-\theta_{m}$ (recall that $\gamma^{\varepsilon}$ is the geometrical parameter defined in (5)).

On the considered rectangle we use an approximation of the Euler-Bernoulli displacement adapted to the displacements we just fixed on the balls $B_{I, r}^{\varepsilon}$ and $B_{I+p, s}^{\varepsilon}$. Indeed this displacement is known to be almost optimal from the energetic point of view. We set, decomposing $x=\varepsilon x_{1} \tilde{e}^{1}+\varepsilon^{2} x_{2} \tilde{e}^{2}$,

$$
u^{\varepsilon}(x):= \begin{cases}U_{I, r}^{\varepsilon}+\theta_{I, r}^{\varepsilon}\left(x+\varepsilon \frac{\ell_{p, r, s}}{2} \tilde{e}^{1}\right)^{\perp} & \text { if } x_{1}<-\gamma^{\varepsilon \frac{\ell_{p, r, s}}{2}}, \\ U_{I+p, s}^{\varepsilon}+\theta_{I+p, s}^{\varepsilon}\left(x-\varepsilon \frac{\ell_{p, r, s}}{2} \tilde{e}^{1}\right)^{\perp} & \text { if } x_{1}>+\gamma^{\varepsilon} \frac{\ell_{p, r, s}}{2}, \\ U_{m}+\theta_{m} x^{\perp}+u_{1}^{\varepsilon}(x) \tilde{e}^{1}+u_{2}^{\varepsilon}(x) \tilde{e}^{2} & \text { otherwise, }\end{cases}
$$

with

$$
\begin{aligned}
u_{1}^{\varepsilon}(x):=\left(U_{1}^{+}-U_{1}^{-}\right) \frac{x_{1}}{\gamma^{\varepsilon} \ell_{p, r, s}}-\left(\frac{12 x_{1}^{2}}{\left(\gamma^{\varepsilon}\right)^{2}}\left(\theta^{+}+\theta^{-}\right)+\frac{4 \ell_{p, r, s} x_{1}}{\gamma^{\varepsilon}}\left(\theta^{+}-\theta^{-}\right)-\ell_{p, r, s}^{2}\left(\theta^{+}+\theta^{-}\right)\right) \frac{\varepsilon^{2} x_{2}}{4 \ell_{p, r, s}^{2}}, \\
\begin{aligned}
u_{2}^{\varepsilon}(x):=\varepsilon \frac{\gamma^{\varepsilon}}{8 \ell_{p, r, s}^{2}}\left(\frac{2 x_{1}}{\gamma^{\varepsilon}}\left(\theta^{+}+\theta^{-}\right)+\ell_{p, r, s}\left(\theta^{+}-\theta^{-}\right)\right)\left(\frac{4 x_{1}^{2}}{\gamma^{\varepsilon}}-\ell_{p, r, s}^{2}\right) \\
-\varepsilon \frac{\gamma^{\varepsilon} \nu \varphi^{\varepsilon}\left(x_{1}\right)}{\ell_{p, r, s}^{2}}\left(\ell_{p, r, s}\left(U_{1}^{+}-U_{1}^{-}\right) x_{2}-\left(\frac{6 x_{1}}{\gamma^{\varepsilon}}\left(\theta^{+}+\theta^{-}\right)+\ell_{p, r, s}\left(\theta^{+}-\theta^{-}\right)\right) \frac{\varepsilon^{2} x_{2}^{2}}{2}\right)
\end{aligned}
\end{aligned}
$$

where $\varphi^{\varepsilon}$ stands for the continuous piece-wise affine function defined by

$$
\varphi^{\varepsilon}(t)= \begin{cases}1 & \text { if }|t|<\left(2 \gamma^{\varepsilon}-1\right) \frac{\ell_{p, r, s}}{2} \\ 0 & \text { if }|t|>\gamma^{\varepsilon} \frac{\ell_{p, r, s}}{2} .\end{cases}
$$

The derivative of $\varphi^{\varepsilon}$ is bounded by

$$
\left|\left(\varphi^{\varepsilon}\right)^{\prime}(t)\right| \leq \frac{2}{\left(1-\gamma^{\varepsilon}\right) \ell_{p, r, s}} \leq \frac{2 \tan \left(\alpha_{\min } / 2\right)}{\varepsilon \beta}
$$

Note that the function $u^{\varepsilon}$ is multiply-defined in the vicinity of each node $y_{I, r}^{\varepsilon}$. These definitions are coherent since the definitions of $u^{\varepsilon}$ on the ball and on the rectangle coincide on $B_{I, r}^{\varepsilon} \cap R_{I, p, r, s}^{\varepsilon}$. Moreover the definitions on two rectangles $R_{I, p, r, s}^{\varepsilon}$ and $R_{I, q, r, t}^{\varepsilon}$ sharing the same end-point $y_{I, r}^{\varepsilon}$ both coincide with $U_{I, r}^{\varepsilon}+\theta_{I, r}^{\varepsilon}\left(x-y_{I, r}^{\varepsilon}\right)^{\perp}$ on $R_{I, p, r, s}^{\varepsilon} \cap R_{I, q, r, t}^{\varepsilon}$ owing to our choice (5) of $\gamma^{\varepsilon}$.

On the other hand, when $x_{1}=-\frac{\ell_{p, r, s} \gamma^{\varepsilon}}{2}$ or $x_{1}=+\frac{\ell_{p, r, s} \gamma^{\varepsilon}}{2}$ expression 25 reads

$$
\left.u^{\varepsilon}(x)=U_{I, r}^{\varepsilon}+\theta_{I, r}^{\varepsilon}\left(\varepsilon \frac{\left(1-\gamma^{\varepsilon}\right) \ell_{p, r, s}}{2}\right) \tilde{e}^{1}+\varepsilon^{2} x_{2} \tilde{e}^{2}\right)^{\perp}
$$

or

$$
u^{\varepsilon}(x)=U_{I+p, s}^{\varepsilon}+\theta_{I+p, s}^{\varepsilon}\left(-\varepsilon \frac{\left(1-\gamma^{\varepsilon}\right) \ell_{p, r, s}}{2} \tilde{e}^{1}+\varepsilon^{2} x_{2} \tilde{e}^{2}\right)^{\perp} .
$$


Thus continuity of $u^{\varepsilon}$ is ensured inside each rectangle. Up to now the function $u^{\varepsilon}$ has been defined as a continuous piece-wise $C^{1}$ function on the union $\tilde{\Omega}^{\varepsilon}$ of all balls $B_{I, r}^{\varepsilon}$ and all rectangles $R_{I, p, r, s}^{\varepsilon}$ for $(p, r, s) \in \tilde{\mathscr{A}}$.

We now estimate the gradient of $u^{\varepsilon}$ on $\tilde{\Omega}^{\varepsilon}$. In a ball or near the end-point in a rectangle we have, using (24), $\left\|\nabla u^{\varepsilon}\right\|=\sqrt{2}\left|\theta_{I, r}^{\varepsilon}\right| \leq C M$. On the central part of each rectangle, differentiating equation (26) we get

$$
\begin{aligned}
& \left(\nabla u^{\varepsilon}\right)_{1,1}(x)=\frac{1}{\varepsilon\left(\gamma^{\varepsilon}\right)^{2} \ell_{p, r, s}^{2}}\left(\gamma^{\varepsilon} \ell_{p, r, s}\left(U_{1}^{+}-U_{1}^{-}\right)-\left(6 x_{1}\left(\theta^{+}+\theta^{-}\right)+\gamma^{\varepsilon} \ell_{p, r, s}\left(\theta^{+}-\theta^{-}\right)\right) \varepsilon^{2} x_{2}\right) \\
& \left(\nabla u^{\varepsilon}\right)_{1,2}(x)=-\theta_{m}-\left(\left(\frac{3 x_{1}^{2}}{\left(\gamma^{\varepsilon}\right)^{2} \ell_{p, r, s}^{2}}-\frac{1}{4}\right)\left(\theta^{+}+\theta^{-}\right)+\frac{x_{1}}{\gamma^{\varepsilon} \ell_{p, r, s}}\left(\theta^{+}-\theta^{-}\right)\right) .
\end{aligned}
$$

Differentiating equation (27) we get

$$
\begin{gathered}
\left(\nabla u^{\varepsilon}\right)_{2,1}(x)=\theta_{m}+\left(\theta^{+}+\theta^{-}\right)\left(\frac{3 x_{1}^{2}}{\gamma^{\varepsilon} \ell_{p, r, s}^{2}}-\frac{1}{4}\right)+\frac{x_{1}}{\gamma^{\varepsilon} \ell_{p, r, s}}\left(\theta^{+}-\theta^{-}\right)+\varepsilon^{2}\left(\theta^{+}+\theta^{-}\right) \frac{3 \nu \varphi^{\varepsilon}\left(x_{1}\right)}{\ell_{p, r, s}^{2}} x_{2}^{2} \\
-\frac{\gamma^{\varepsilon} \nu \varphi^{\varepsilon \prime}\left(x_{1}\right)}{\ell_{p, r, s}^{2}}\left(\ell_{p, r, s}\left(U_{1}^{+}-U_{1}^{-}\right) x_{2}-\left(\frac{6 x_{1}}{\gamma^{\varepsilon}}\left(\theta^{+}+\theta^{-}\right)+\ell_{p, r, s}\left(\theta^{+}-\theta^{-}\right)\right) \frac{\varepsilon^{2} x_{2}^{2}}{2}\right), \\
\left(\nabla u^{\varepsilon}\right)_{2,2}(x)=-\frac{1}{\varepsilon} \frac{\nu \varphi^{\varepsilon}\left(x_{1}\right)}{\ell_{p, r, s}^{2}}\left(\gamma^{\varepsilon} \ell_{p, r, s}\left(U_{1}^{+}-U_{1}^{-}\right)-\left(6 x_{1}\left(\theta^{+}+\theta^{-}\right)+\gamma^{\varepsilon} \ell_{p, r, s}\left(\theta^{+}-\theta^{-}\right)\right) \varepsilon^{2} x_{2}\right) .
\end{gathered}
$$

Using (28) and estimations (24), simple application of triangle inequality shows that the components of $\nabla u^{\varepsilon}$ on the rectangle $R_{I, p, r, s}^{\varepsilon}$ are all bounded by $C M$, where the constant $C$ depends only on the Poisson ratio $\nu$ and the global geometrical parameters $\beta, \ell_{\min }, \ell_{\max }$. We already noticed in Section 2.1 that we can deduce from this estimation that $u^{\varepsilon}$ is $k_{L}$-Lipschitz with $k_{L}:=C M \sqrt{\frac{2}{1-\cos \left(\alpha_{m i n}\right)}}$ on this domain. Hence, owing to Kirszbraun theorem, we know that there exists a $k_{L}$-Lipschitz extension over the whole domain $\Omega$.

- On the complementary set: We simply set $u^{\varepsilon}$ equal to this $k_{L}$-Lipschitz extension.

Let us now check that $u^{\varepsilon}$ converges to $u$ and has the desired limit energy. On each cell $C_{I}^{\varepsilon}$, using the fact that

$$
u^{\varepsilon}\left(y_{I, r}^{\varepsilon}\right)=U_{I, r}^{\varepsilon}=u\left(y_{I, r}^{\varepsilon}\right)+\varepsilon v_{1}\left(y_{I, r}^{\varepsilon}\right)+\varepsilon^{2} w_{1}\left(y_{I, r}^{\varepsilon}\right)
$$

and bounds (24), we have

$$
\begin{aligned}
\left\|u^{\varepsilon}(x)-u(x)\right\| & \leq\left\|u^{\varepsilon}(x)-u^{\varepsilon}\left(y_{I, r}^{\varepsilon}\right)\right\|+\left\|u\left(y_{I, r}^{\varepsilon}\right)+\varepsilon v_{1}\left(y_{I, r}^{\varepsilon}\right)+\varepsilon^{2} w_{1}\left(y_{I, r}^{\varepsilon}\right)-u(x)\right\| \\
& \leq\left\|u^{\varepsilon}(x)-u^{\varepsilon}\left(y_{I, r}^{\varepsilon}\right)\right\|+\left\|u\left(y_{I, r}^{\varepsilon}\right)-u(x)\right\|+\left\|\varepsilon v_{1}\left(y_{I, r}^{\varepsilon}\right)+\varepsilon^{2} w_{1}\left(y_{I, r}^{\varepsilon}\right)\right\| \\
& \leq k_{L} \sqrt{2} \varepsilon+M \sqrt{2} \varepsilon+M \varepsilon .
\end{aligned}
$$

Hence $u^{\varepsilon}$ converges strongly to $u$ in $\mathrm{L}^{2}(\Omega)$. In order to evaluate $\mathscr{E}_{\varepsilon}\left(u^{\varepsilon}\right)$, we first remark that the energy concentrated on $\Omega \backslash \Omega^{\varepsilon}$ tends to zero. Indeed we have, using (7),

$$
\int_{\Omega \backslash \Omega^{\varepsilon}} Y(x)\left(\frac{1}{2(1+\nu)}\left\|e\left(u^{\varepsilon}\right)\right\|^{2}+\frac{\nu}{2\left(1-\nu^{2}\right)}\left(\operatorname{tr}\left(e\left(u^{\varepsilon}\right)\right)\right)^{2}\right) d x \leq Y_{1} \varepsilon^{a} \int_{\Omega \backslash \Omega^{\varepsilon}} \frac{1}{4}\left\|e\left(u^{\varepsilon}\right)\right\|^{2} d x \leq Y_{1} \varepsilon^{a} k_{L}^{2} .
$$


Moreover, in the vicinity of nodes $y_{I, r}^{\varepsilon}$ (that is on the balls $B_{I, r}^{\varepsilon}$ and on the parts of the rectangles where $\left.2\left|x_{1}\right|>\left(1-\gamma^{\varepsilon}\right) \ell_{p, r, s}\right)$ the displacement $u^{\varepsilon}$ coincides with rigid motions. Thus $e\left(u^{\varepsilon}\right)$ vanishes and no energy is concentrated there.

It remains to estimate the energy concentrated on the "central part" (that is where $2\left|x_{1}\right|<$ $\left.\left(1-\gamma^{\varepsilon}\right) \ell_{p, r, s}\right)$ of the rectangles $R_{I, p, r, s}^{\varepsilon}$ for all $(p, r, s) \in \mathscr{A}$. We begin by noticing that we can have a better estimation of $\left(U_{I+p, s}^{\varepsilon}-U_{I, r}^{\varepsilon, s}\right) \cdot \tau_{p, r, s}$ when $(p, r, s) \in \mathscr{A}$. Indeed, from $\bar{E}\left(v, \eta_{u}\right)=0$ which reads

$$
\int_{\Omega} \sum_{(p, r, s) \in \mathscr{A}} \frac{Y_{0} \beta}{\ell_{p, r, s}}\left(\left(v_{s}(x)-v_{r}(x)+\nabla u(x) \cdot \mathbf{p}\right) \cdot \tau_{p, r, s}\right)^{2} d x=0,
$$

we deduce that, for any $x \in \Omega$ and any $(p, r, s) \in \mathscr{A}$,

$$
\left(v_{s}(x)-v_{r}(x)+\nabla u(x) \cdot \mathbf{p}\right) \cdot \tau_{p, r, s}=0 .
$$

As a consequence,

$$
\begin{aligned}
& \left(U_{I+p, s}^{\varepsilon}-U_{I, r}^{\varepsilon}\right) \cdot \tau_{p, r, s}=\left(u\left(y_{I+p}^{\varepsilon}\right)-u\left(y_{I}^{\varepsilon}\right)+\varepsilon\left(v_{s}\left(y_{I+p}^{\varepsilon}\right)-v_{r}\left(y_{I}^{\varepsilon}\right)\right)+\varepsilon^{2}\left(w_{s}\left(y_{I+p}^{\varepsilon}\right)-w_{r}\left(y_{I}^{\varepsilon}\right)\right)\right) \cdot \tau_{p, r, s} \\
& \quad=\left(u\left(y_{I+p}^{\varepsilon}\right)-u\left(y_{I}^{\varepsilon}\right)-\nabla u\left(y_{I}^{\varepsilon}\right) \cdot \varepsilon \mathbf{p}+\varepsilon\left(v_{s}\left(y_{I+p}^{\varepsilon}\right)-v_{s}\left(y_{I}^{\varepsilon}\right)\right)+\varepsilon^{2}\left(w_{s}\left(y_{I+p}^{\varepsilon}\right)-w_{r}\left(y_{I}^{\varepsilon}\right)\right)\right) \cdot \tau_{p, r, s}
\end{aligned}
$$

and thus, using Taylor expansions,

$$
\left|\left(U_{I+p, s}^{\varepsilon}-U_{I, r}^{\varepsilon}\right) \cdot \tau_{p, r, s}\right| \leq \varepsilon^{2} C M
$$

or still more precisely, using further Taylor expansions,

$$
\left|\left(U_{I+p, s}^{\varepsilon}-U_{I, r}^{\varepsilon}-\frac{1}{2} \nabla \nabla u\left(y_{I}^{\varepsilon}\right):\left(\varepsilon^{2} \mathbf{p} \otimes \mathbf{p}\right)-\varepsilon \nabla v_{s}\left(y_{I}^{\varepsilon}\right) \cdot \varepsilon \mathbf{p}-\varepsilon^{2}\left(w_{s}\left(y_{I}^{\varepsilon}\right)-w_{r}\left(y_{I}^{\varepsilon}\right)\right)\right) \cdot \tau_{p, r, s}\right| \leq \varepsilon^{3} C M .
$$

In the central part of each rectangle $R_{I, p, r, s}^{\varepsilon}$ corresponding to an edge $(p, r, s)$ in $\mathscr{A}$, we have already computed in equations (29) and (32) the strain components $e_{1,1}\left(u^{\varepsilon}\right)$ and $e_{2,2}\left(u^{\varepsilon}\right)$. From (30) and (31) we get

$$
\begin{aligned}
e_{1,2}\left(u^{\varepsilon}\right)(x)=\varepsilon^{2}\left(\theta^{+}+\right. & \left.\theta^{-}\right) \frac{3 \nu \varphi^{\varepsilon}\left(x_{1}\right)}{\ell_{p, r, s}^{2}} x_{2}^{2} \\
& -\frac{\gamma^{\varepsilon} \nu\left(\varphi^{\varepsilon}\right)^{\prime}\left(x_{1}\right)}{\ell_{p, r, s}^{2}}\left(\ell_{p, r, s}\left(U_{1}^{+}-U_{1}^{-}\right) x_{2}-\left(\frac{6 x_{1}}{\gamma^{\varepsilon}}\left(\theta^{+}+\theta^{-}\right)+\ell_{p, r, s}\left(\theta^{+}-\theta^{-}\right)\right) \frac{\varepsilon^{2} x_{2}^{2}}{2}\right) .
\end{aligned}
$$

Using (34), that is $\left|U_{1}^{+}-U_{1}^{-}\right|=\left|\left(U_{I+p, s}^{\varepsilon}-U_{I, r}^{\varepsilon}\right) \cdot \tau_{p, r, s}\right| \leq \varepsilon^{2} C M$, we get

$$
\left\|e_{1,2}\left(u^{\varepsilon}\right)(x)\right\| \leq \varepsilon^{2} C M+\varepsilon^{2}\left|\left(\varphi^{\varepsilon}\right)^{\prime}\left(x_{1}\right)\right| C M
$$

which implies

$$
\left\|e_{1,2}\left(u^{\varepsilon}\right)(x)\right\| \leq \varepsilon C M .
$$

We also notice that, on the considered sets, $e_{2,2}\left(u^{\varepsilon}\right)=-\varphi^{\varepsilon}\left(x_{1}\right) \nu e_{1,1}\left(u^{\varepsilon}\right)$. Thus

$$
\begin{aligned}
\frac{1}{2(1+\nu)}\left(\left(e_{1,1}\left(u^{\varepsilon}\right)\right)^{2}+\left(e_{2,2}\left(u^{\varepsilon}\right)\right)^{2}\right)+\frac{\nu}{2\left(1-\nu^{2}\right)}\left(e_{1,1}\left(u^{\varepsilon}\right)+e_{2,2}\left(u^{\varepsilon}\right)\right)^{2} & \\
& =\frac{1}{2}\left(1+\frac{\nu^{2}}{1-\nu^{2}}\left(1-\varphi^{\varepsilon}\left(x_{1}\right)\right)^{2}\right)\left(e_{1,1}\left(u^{\varepsilon}\right)\right)^{2}
\end{aligned}
$$


which together with 36 gives the following bound for the energy density:

$$
\begin{aligned}
& \frac{1}{2(1+\nu)}\left\|e\left(u^{\varepsilon}\right)\right\|^{2}+\frac{\nu}{2\left(1-\nu^{2}\right)}\left(\operatorname{tr}\left(e\left(u^{\varepsilon}\right)\right)\right)^{2} \\
& \quad \leq \frac{1}{2}\left(1+\frac{\nu^{2}}{1-\nu^{2}}\left(1-\varphi^{\varepsilon}\left(x_{1}\right)\right)^{2}\right)\left(e_{1,1}\left(u^{\varepsilon}\right)\right)^{2}+\varepsilon^{4} C M^{2}+\varepsilon^{4}\left|\varphi^{\varepsilon \prime}\left(x_{1}\right)\right|^{2} C M^{2}
\end{aligned}
$$

The energy concentrated in the central part of the rectangle, namely

$$
\left.\int_{-\frac{\gamma^{\varepsilon} \ell_{p, r, s}}{2}}^{+\frac{\gamma^{\varepsilon} \ell_{p, r, s}}{2}} Y(x)\left(\frac{1}{2(1+\nu)}\left\|e\left(u^{\varepsilon}\right)\right\|^{2}+\frac{\nu}{2\left(1-\nu^{2}\right)}\left(\operatorname{tr}\left(e\left(u^{\varepsilon}\right)\right)\right)^{2}\right) \varepsilon^{2} d x_{2}\right) \varepsilon d x_{1},
$$

can be estimated by considering separately the transition layers $\frac{\left(2 \gamma^{\varepsilon}-1\right) \ell_{p, r, s}}{2}<\left|x_{1}\right|<\frac{\gamma^{\varepsilon} \ell_{p, r, s}}{2}$ where $\varphi^{\varepsilon}$ varies and the "very central" part $\left|x_{1}\right|<\frac{\left.2 \gamma^{\varepsilon}-1\right) \ell_{p, r, s}}{2}$ where $\varphi^{\varepsilon}=1$. As far as the transition layers are concerned, owing again to (34), we can use the fact that $\left|e_{1,1}\right| \leq \varepsilon k_{L} M$ and thus that the energy density is bounded by $\varepsilon^{2} C M^{2} Y$. The integral over these layers is then bounded by $C M^{2} Y \varepsilon^{5}\left(1-\gamma^{\varepsilon}\right) \beta=\varepsilon^{2} C M^{2} Y_{0}\left(1-\gamma^{\varepsilon}\right)$. The sum over all rectangles is bounded by $C M^{2} Y_{0}\left(1-\gamma^{\varepsilon}\right)$ which tends to zero.

It remains to estimate the energy of the "very central" part:

$$
\begin{aligned}
& E_{I, p, r, s}=\int_{-\frac{\left(2 \gamma^{\varepsilon}-1\right) \ell_{p, r, s}}{2}}^{+\frac{\left(2 \gamma^{\varepsilon}-1\right) \ell_{p, r, s}}{2}}\left(\int_{-\frac{\beta}{2}}^{+\frac{\beta}{2}} Y(x)\left(\frac{1}{2(1+\nu)}\left\|e\left(u^{\varepsilon}\right)\right\|^{2}+\frac{\nu}{2\left(1-\nu^{2}\right)}\left(\operatorname{tr}\left(e\left(u^{\varepsilon}\right)\right)\right)^{2}\right) \varepsilon^{2} d x_{2}\right) \varepsilon d x_{1}, \\
& \leq \int_{-\frac{\left(2 \gamma^{\varepsilon}-1\right) \ell_{p, r, s}}{2}}^{+\frac{\left(2 \gamma^{\varepsilon}-1\right) \ell_{p, r, s}}{2}}\left(\int_{-\frac{\beta}{2}}^{+\frac{\beta}{2}}\left(\frac{Y_{0}}{2}\left(e_{1,1}\left(u^{\varepsilon}\right)\right)^{2}+\varepsilon^{4} C M^{2}\right) d x_{2}\right) d x_{1} \\
& \leq \frac{Y_{0}}{2} \int_{-\frac{\left(2 \gamma^{\varepsilon}-1\right) \ell_{p, r, s}}{2}}^{+\frac{\left(2 \gamma^{\varepsilon}-1\right) \ell_{p, r, s}}{2}}\left(\int_{-\frac{\beta}{2}}^{+\frac{\beta}{2}}\left(\left(e_{1,1}\left(u^{\varepsilon}\right)\right)^{2}\right) d x_{2}\right) d x_{1}+\varepsilon^{4} C M^{2} \beta \ell_{p, r, s} \\
& \leq \frac{Y_{0}}{2 \varepsilon^{2}\left(\gamma^{\varepsilon}\right)^{4} \ell_{p, r, s}^{4}} \int_{-\frac{\ell_{p, r, s}}{2}}^{+\frac{\ell_{p, r, s}}{2}}\left(\int _ { - \frac { \beta } { 2 } } ^ { + \frac { \beta } { 2 } } \left(\gamma^{\varepsilon} \ell_{p, r, s}\left(U_{1}^{+}-U_{1}^{-}\right)\right.\right. \\
& \left.\left.-\left(6 x_{1}\left(\theta^{+}+\theta^{-}\right)+\gamma^{\varepsilon} \ell_{p, r, s}\left(\theta^{+}-\theta^{-}\right)\right) \varepsilon^{2} x_{2}\right)^{2} d x_{2}\right) d x_{1}+\varepsilon^{4} C M^{2} \\
& \leq \frac{Y_{0}}{2 \varepsilon^{2}\left(\gamma^{\varepsilon}\right)^{4} \ell_{p, r, s}^{4}} \int_{-\frac{\ell_{p, r, s}}{2}}^{+\frac{\ell_{p, r, s}}{2}}\left(\int _ { - \frac { \beta } { 2 } } ^ { + \frac { \beta } { 2 } } \left(\ell_{p, r, s}^{2}\left(U_{1}^{+}-U_{1}^{-}\right)^{2}\right.\right. \\
& \left.\left.+\left(36 x_{1}^{2}\left(\theta^{+}+\theta^{-}\right)^{2}+\ell_{p, r, s}^{2}\left(\theta^{+}-\theta^{-}\right)^{2}\right) \varepsilon^{4} x_{2}^{2}\right) d x_{2}\right) d x_{1}+\varepsilon^{4} C M^{2} \\
& \leq \frac{\varepsilon^{2} Y_{0} \beta}{2\left(\gamma^{\varepsilon}\right)^{4} \ell_{p, r, s}}\left(\left(\frac{U_{1}^{+}-U_{1}^{-}}{\varepsilon^{2}}\right)^{2}+\frac{\beta^{2}}{12}\left(3\left(\theta^{+}+\theta^{-}\right)^{2}+\left(\theta^{+}-\theta^{-}\right)^{2}\right)\right)+\varepsilon^{4} C M^{2} \\
& \leq \frac{\varepsilon^{2} Y_{0} \beta}{2\left(\gamma^{\varepsilon}\right)^{4} \ell_{p, r, s}}\left(\left(\frac{\left(U_{I+p, s}^{\varepsilon}-U_{I, r}^{\varepsilon}\right) \cdot \tau_{p, r, s}}{\varepsilon^{2}}\right)^{2}+\right. \\
& \left.\frac{\beta^{2}}{12}\left(\frac{3}{\left(\gamma^{\varepsilon}\right)^{2}}\left(\theta_{I+p, s}^{\varepsilon}+\theta_{I, r}^{\varepsilon}-2 \frac{\left(U_{I+p, s}^{\varepsilon}-U_{I, r}^{\varepsilon}\right) \cdot \tau_{p, r, s}^{\perp}}{\varepsilon \ell_{p, r, s}}\right)^{2}+\left(\theta_{I+p, s}^{\varepsilon}-\theta_{I, r}^{\varepsilon}\right)^{2}\right)\right)+\varepsilon^{4} C M^{2} .
\end{aligned}
$$


Let us define the $C^{\infty}$ function $\Phi_{p, r, s}$ by setting, for any $x$ in $\Omega$,

$$
\Phi_{p, r, s}(x):=\left(\frac{1}{2} \nabla \nabla u(x) \cdot \mathbf{p} \cdot \mathbf{p}+\nabla v_{s}(x) \cdot \varepsilon \mathbf{p}+\left(w_{s}(x)-w_{r}(x)\right)\right) \cdot \tau_{p, r, s}
$$

so that bound (35) simply reads

$$
\left|\left(U_{I+p, s}^{\varepsilon}-U_{I, r}^{\varepsilon}\right) \cdot \tau_{p, r, s}-\varepsilon^{2} \Phi_{p, r, s}\left(y_{I}^{\varepsilon}\right)\right| \leq \varepsilon^{3} C M .
$$

As $\Phi_{p, r, s}$ is clearly bounded $\left(\left\|\Phi_{p, r, s}\right\|_{\mathrm{L}^{\infty}(\Omega)}<C M\right)$, this inequality implies

$$
\left(\frac{\left(U_{I+p, s}^{\varepsilon}-U_{I, r}^{\varepsilon}\right) \cdot \tau_{p, r, s}}{\varepsilon^{2}}\right)^{2} \leq\left(\Phi_{p, r, s}\left(y_{I}^{\varepsilon}\right)\right)^{2}+\varepsilon C M^{2}
$$

In a similar way, using Taylor expansions,

$$
\begin{aligned}
\mid \theta_{I+p, s}^{\varepsilon} & +\theta_{I, r}^{\varepsilon}-2 \frac{\left(U_{I+p, s}^{\varepsilon}-U_{I, r}^{\varepsilon}\right) \cdot \tau_{p, r, s}^{\perp}}{\varepsilon \ell_{p, r, s}} \mid \\
& =\left|\theta_{s}^{\varepsilon}\left(y_{I}^{\varepsilon}\right)+\theta_{r}^{\varepsilon}\left(y_{I}^{\varepsilon}\right)-2 \frac{\left(u\left(y_{I+p}^{\varepsilon}\right)-u\left(y_{I}^{\varepsilon}\right)+\varepsilon\left(v_{s}\left(y_{I}^{\varepsilon}\right)-v_{r}\left(y_{I}^{\varepsilon}\right)\right)+\varepsilon^{2}\left(w_{s}\left(y_{I}^{\varepsilon}\right)-w_{r}\left(y_{I}^{\varepsilon}\right)\right)\right) \cdot \tau_{p, r, s}^{\perp}}{\varepsilon \ell_{p, r, s}}\right| \\
& \leq\left|\theta_{s}^{\varepsilon}\left(y_{I}^{\varepsilon}\right)+\theta_{r}^{\varepsilon}\left(y_{I}^{\varepsilon}\right)-2 \frac{\left.\left(\nabla u\left(y_{I}^{\varepsilon}\right) \cdot \mathbf{p}+v_{s}\left(y_{I}^{\varepsilon}\right)-v_{r}\left(y_{I}^{\varepsilon}\right)\right)\right) \cdot \tau_{p, r, s}^{\perp}}{\ell_{p, r, s}}\right|+\varepsilon C M \\
& \leq\left|\Psi_{p, r, s}\left(y_{I}^{\varepsilon}\right)\right|+\varepsilon C M,
\end{aligned}
$$

where we introduced the function $\left.\Psi_{p, r, s}(x):=\theta_{s}^{\varepsilon}(x)+\theta_{r}^{\varepsilon}(x)-\frac{2}{\ell_{p, r, s}}\left(\nabla u(x) \cdot \mathbf{p}+v_{s}(x)-v_{r}(x)\right)\right) \cdot \tau_{p, r, s}^{\perp}$. This implies

$$
\left(\theta_{I+p, s}^{\varepsilon}+\theta_{I, r}^{\varepsilon}-2 \frac{\left(U_{I+p, s}^{\varepsilon}-U_{I, r}^{\varepsilon}\right) \cdot \tau_{p, r, s}^{\perp}}{\varepsilon \ell_{p, r, s}}\right)^{2} \leq\left(\Psi_{p, r, s}\left(y_{I}^{\varepsilon}\right)\right)^{2}+\varepsilon C M^{2}
$$

Finally,

which implies

$$
\left|\theta_{I+p, s}^{\varepsilon}-\theta_{I, r}^{\varepsilon}\right|=\left|\theta_{s}\left(y_{I+p}^{\varepsilon}\right)-\theta_{r}\left(y_{I}^{\varepsilon}\right)\right| \leq\left|\theta_{s}\left(y_{I}^{\varepsilon}\right)-\theta_{r}\left(y_{I}^{\varepsilon}\right)\right|+\varepsilon C M
$$

$$
\left(\theta_{I+p, s}^{\varepsilon}-\theta_{I, r}^{\varepsilon}\right)^{2} \leq\left(\left(\theta_{s}-\theta_{r}\right)\left(y_{I}^{\varepsilon}\right)\right)^{2}+\varepsilon C M^{2} .
$$

Collecting estimations (38), (39) and (40), we obtain

$$
E_{I, p, r, s} \leq \frac{\varepsilon^{2} Y_{0} \beta}{2\left(\gamma^{\varepsilon}\right)^{4} \ell_{p, r, s}}\left(\left(\Phi_{p, r, s}\left(y_{I}^{\varepsilon}\right)\right)^{2}+\frac{\beta^{2}}{12}\left(\frac{3}{\left(\gamma^{\varepsilon}\right)^{2}}\left(\Psi_{p, r, s}\left(y_{I}^{\varepsilon}\right)\right)^{2}+\left(\left(\theta_{s}-\theta_{r}\right)\left(y_{I}^{\varepsilon}\right)\right)^{2}\right)\right)+\varepsilon^{3} C M^{2} .
$$

Summing over all $I$ corresponds to a Riemann summation of a continuous function and we get

$$
\sum_{I \in \mathscr{I} \varepsilon} E_{I, p, r, s} \leq \int_{\Omega} \frac{Y_{0} \beta}{2\left(\gamma^{\varepsilon}\right)^{4} \ell_{p, r, s}}\left(\left(\Phi_{p, r, s}(x)\right)^{2}+\frac{\beta^{2}}{12}\left(\frac{3}{\left(\gamma^{\varepsilon}\right)^{2}}\left(\Psi_{p, r, s}(x)\right)^{2}+\left(\left(\theta_{s}-\theta_{r}\right)(x)\right)^{2}\right)\right) d x+\varepsilon C M^{2} .
$$

Summing over all $(p, r, s)$ in $\mathscr{A}$ leads to the desired result when replacing functions $\Phi_{p, r, s}$ and $\Psi_{p, r, s}$ by their expressions:

$$
\lim _{\varepsilon \rightarrow 0} \mathscr{E}_{\varepsilon}\left(u^{\varepsilon}\right)=\lim _{\varepsilon \rightarrow 0} \sum_{(p, r, s) \in \mathscr{A}} \sum_{I \in \mathscr{I} \varepsilon} E_{I, p, r, s}=\bar{E}\left(w, \xi_{u, v}\right)+\bar{F}\left(v, \eta_{u}, \theta\right)=\mathscr{E}(u) .
$$




\section{Conclusion}

In this work, we extended the homogenization result established in [1] for periodic elastic lattices to the case of a non-degenerate elastic material reinforced by very stiff fibers arranged along such lattices. We thus enable comparison with homogenization results established for non-degenerate materials and, in particular, we justify the comparisons made in [21]. More standard external forces applied in the whole domain can now be taken into account.

Note that we have only considered here free boundary conditions. Imposing a Dirichlet boundary condition or imposing a line density of forces (non homogeneous Neumann boundary condition) along the boundary of the domain would need a more sophisticated study of the capacity of the lattice. It is the object of future works.

\section{Acknowledgement}

The authors acknowledge the support of the French Agence Nationale de la Recherche (ANR), under grant ANR-17-CE08-0039 (project ArchiMatHOS).

\section{Appendix}

Lemma 1 states the existence of a constant $C$ such that, for any $u \in \mathrm{H}^{1}(\Omega)$,

$$
\left\|u-\tilde{u}_{s}^{\varepsilon}\right\|_{\mathrm{L}^{2}(\Omega)}^{2} \leq C \varepsilon^{2}|\ln (\varepsilon)|\|\nabla u\|_{\mathrm{L}^{2}(\Omega)}^{2} .
$$

where the quantities $\bar{u}_{I, s}^{\varepsilon}$ and the piece-wise constant function $\tilde{u}_{s}^{\varepsilon}(x)$ are associated to $u$ by

$$
\bar{u}_{I, s}^{\varepsilon}:={\underset{B}{B_{I, s}^{\varepsilon}}}_{f} u(x) d x \quad \text { and } \quad \tilde{u}_{s}^{\varepsilon}(x):=\sum_{I \in \mathscr{I}^{\varepsilon}} \bar{u}_{I, s}^{\varepsilon} 1_{C_{I}^{\varepsilon}}(x) .
$$

Proof. In order to prove this result, we define another auxiliary function $\tilde{\tilde{u}}_{s}^{\varepsilon}$. For any $s \in\{1, \ldots, K\}$ and $I=(i, j) \in \mathscr{I}^{\varepsilon}$, we introduce the annulus $D_{i, s}^{\varepsilon}:=\left\{x: k \varepsilon<\left\|x-y_{I, s}^{\varepsilon}\right\|<2 k \varepsilon\right\}$ (the parameter $k$ is chosen in such a way that $D_{i, s}^{\varepsilon} \subset C_{I}^{\varepsilon}$ and $\varepsilon$ is small enough for ensuring $\varepsilon^{2}<k \varepsilon$ ) and we define the quantities $\overline{\bar{u}}_{I, s}^{\varepsilon}$ and the piece-wise constant function $\tilde{\tilde{u}}_{s}^{\varepsilon}(x)$ are associated to $u$ by $\overline{\bar{u}}_{I, s}^{\varepsilon}:=f_{D_{I, s}^{\varepsilon}} u(x) d x$ and

$$
\tilde{\tilde{u}}_{s}^{\varepsilon}(x):=\sum_{I \in \mathscr{I}^{\varepsilon}} \overline{\bar{u}}_{I, s}^{\varepsilon} 1_{C_{I}^{\varepsilon}}(x) .
$$

We first remark that there exists a constant $C$ independent on $I$ such that

$$
\int_{C_{I}^{1}}\left\|u-\int_{D_{I, s}^{1}} u d y\right\|^{2} d x \leq C \int_{C_{I}^{1}}\|\nabla u\|^{2} d x
$$

Indeed, assume, by contradiction, that there exists a sequence $u^{n}$ satisfying

$$
\int_{C_{I}^{1}}\left\|u^{n}-f_{D_{I, s}^{1}} u^{n} d y\right\|^{2} d x=1 \quad \text { and } \quad \int_{C_{I}^{1}}\left\|\nabla u^{n}\right\|^{2} d x \rightarrow 0
$$


The function $v^{n}:=u^{n}-f_{D_{I, s}^{1}} u^{n} d y$ is bounded in $\mathrm{H}^{1}\left(C_{I}^{1}\right)$, and thus must converge strongly in $\mathrm{L}^{2}\left(C_{I}^{1}\right)$ to some function $v$ which satisfies $\int_{C_{I}^{1}}\|\nabla v\|^{2} d x=0$. Hence $v$ is constant in $C_{I}^{1}$. As $0=f_{D_{I, s}^{1}} v^{n} \rightarrow f_{D_{I, s}^{1}} v d x$, we know that $v=0$ which is in contradiction with the assumption that $\int_{C_{I}^{1}}\left\|v^{n}\right\|^{2} d x=1$. Inequality (42) is now established. A simple rescaling by factor $\varepsilon$ transforms it into

$$
\int_{C_{I}^{\varepsilon}}\left\|u-f_{D_{I, s}^{\varepsilon}} u d y\right\|^{2} d x \leq C \varepsilon^{2} \int_{C_{I}^{\varepsilon}}\|\nabla u\|^{2} d x .
$$

Summing over all cells gives

$$
\left\|u-\tilde{\tilde{u}}_{s}^{\varepsilon}\right\|_{\mathrm{L}^{2}(\Omega)}^{2} \leq C \varepsilon^{2}\|\nabla u\|_{\mathrm{L}^{2}(\Omega)}^{2} .
$$

Let us now focus on a cell $C_{I}^{\varepsilon}$ and use there the polar coordinates $(\rho, \theta)$ with origin $y_{I, s}^{\varepsilon}$. To any $u \in \mathrm{H}^{1}\left(C_{I}^{\varepsilon}\right)$, let us associate $v(\rho):=\frac{1}{2 \pi} \int_{0}^{2 \pi} u(\rho, \theta) d \theta$. A simple one-dimensional optimization shows that, for any $0<\rho_{1}<\rho_{2}<2 k \varepsilon$,

$$
\int_{\rho_{1}}^{\rho_{2}}\left\|v^{\prime}(\rho)\right\|^{2} \rho d \rho \geq \frac{\left\|v\left(\rho_{2}\right)-v\left(\rho_{1}\right)\right\|^{2}}{\ln \left(\rho_{2}\right)-\ln \left(\rho_{1}\right)}
$$

and thus

$$
\left\|v\left(\rho_{2}\right)-v\left(\rho_{1}\right)\right\|^{2} \leq\left(\ln \left(\rho_{2}\right)-\ln \left(\rho_{1}\right)\right) \int_{0}^{2 k \varepsilon}\left\|v^{\prime}(\rho)\right\|^{2} \rho d \rho .
$$

Now let us take the mean value of both sides of this inequality with respect to the measures $\rho_{1} d \rho_{1}$ and $\rho_{2} d \rho_{2}$ for $\rho_{1} \in\left(0, \varepsilon^{2}\right)$ and $\rho_{2} \in(k \varepsilon, 2 k \varepsilon)$. Noticing that the mean values of $v\left(\rho_{1}\right)$ and $v\left(\rho_{2}\right)$ are nothing else than respectively $\tilde{u}_{I, s}^{\varepsilon}$ and $\bar{u}_{I, s}^{\varepsilon}$ and using Jensen inequality, we get

$$
\left\|\tilde{u}_{I, s}^{\varepsilon}-\bar{u}_{I, s}^{\varepsilon}\right\|^{2} \leq\left(\frac{\int_{k \varepsilon}^{2 k \varepsilon} \ln \left(\rho_{2}\right) \rho_{2} d \rho_{2}}{\int_{k \varepsilon}^{2 k \varepsilon} \rho_{2} d \rho_{2}}-\frac{\int_{0}^{\varepsilon^{2}} \ln \left(\rho_{1}\right) \rho_{1} d \rho_{1}}{\int_{0}^{\varepsilon^{2}} \rho_{1} d \rho_{1}}\right) \int_{0}^{2 k \varepsilon}\left\|v^{\prime}(\rho)\right\|^{2} \rho d \rho
$$

and so

$$
\left\|\overline{\bar{u}}_{I, s}^{\varepsilon}-\bar{u}_{I, s}^{\varepsilon}\right\|^{2} \leq\left(|\ln (\varepsilon)|+\ln (k)+\frac{4}{3} \ln (2)\right) \int_{C_{I}^{\varepsilon}}\|\nabla u\|^{2} \leq C|\ln (\varepsilon)| \int_{C_{I}^{\varepsilon}}\|\nabla u\|^{2} .
$$

Summing over all cells gives

$$
\left\|\tilde{\tilde{u}}_{s}^{\varepsilon}-\tilde{u}_{s}^{\varepsilon}\right\|_{\mathrm{L}^{2}(\Omega)}^{2}=\sum_{I \in \mathscr{I}^{\varepsilon}}\left|C_{I}^{\varepsilon}\right|\left\|\overline{\bar{u}}_{I, s}^{\varepsilon}-\bar{u}_{I, s}^{\varepsilon}\right\|^{2}=\varepsilon^{2} \sum_{I \in \mathscr{I}^{\varepsilon}}\left\|\overline{\bar{u}}_{I, s}^{\varepsilon}-\bar{u}_{I, s}^{\varepsilon}\right\|^{2} \leq C \varepsilon^{2}|\ln (\varepsilon)| \int_{\Omega}\|\nabla u\|^{2} .
$$

Finally inequality (41) results from (44) and (45) by triangle inequality. 


\section{References}

[1] H. Abdoul-Anziz and P. Seppecher. Homogenization of periodic graph-based elastic structures. Journal de l'Ecole polytechnique-Mathématiques, 5:259-288, 2018.

[2] H. Abdoul-Anziz and P. Seppecher. Strain gradient and generalized continua obtained by homogenizing frame lattices. Mathematics and Mechanics of Complex Systems, 6(3):213-250, 2018.

[3] H. Abdoul-Anziz, P. Seppecher, and C. Bellis. Homogenization of frame lattices leading to second gradient models coupling classical strain and strain-gradient terms. Mathematics and Mechanics of Solids, 24(12):3976-3999, 2019.

[4] J.-J. Alibert and A. Della Corte. Second-gradient continua as homogenized limit of pantographic microstructured plates: a rigorous proof. Z. Angew. Math. Phys., 66(5):2855-2870, 2015.

[5] J.-J. Alibert, P. Seppecher, and F. dell'Isola. Truss modular beams with deformation energy depending on higher displacement gradients. Mathematics and Mechanics of Solids, 8(1):51$73,2003$.

[6] G. Allaire. Homogenization and two-scale convergence. SIAM Journal on Mathematical Analysis, 23(6):1482-1518, 1992.

[7] G. Allaire, M. Briane, and M. Vanninathan. A comparison between two-scale asymptotic expansions and bloch wave expansions for the homogenization of periodic structures. SeMA Journal, 73(3):237-259, 2016.

[8] N. S. Bakhvalov and G. Panasenko. Homogenisation: averaging processes in periodic media: mathematical problems in the mechanics of composite materials, volume 36. Springer Science \& Business Media, 2012.

[9] A. Bensoussan, J.-L. Lions, and G. Papanicolau. Asymptotic Analysis for Periodic Structures. North-Holland, Amsterdam, 1978.

[10] G. Bouchitté and M. Bellieud. Homogenization of elliptic problems in a fiber reinforced structure. non local effects. Ann. Scuola Norm. Sup. Cl. Sci., IV(26):407?436, 1998.

[11] G. Bouchitté and M. Bellieud. Homogenization of a soft elastic material reinforced by fibers. Asymptotic Analysis, (32):153-183, 2002.

[12] C. Boutin, F. dell'Isola, I. Giorgio, and L. Placidi. Linear pantographic sheets: Asymptotic micro-macro models identification. MEMOCS, 5(2):127-162, 2017.

[13] A. Braides. Г-convergence for Beginners. Oxford University Press, 2002.

[14] M. Briane and M. Camar-Eddine. Homogenization of two-dimensional elasticity problems with very stiff coefficients. Journal de mathmatiques pures et appliques, 88(6):483-505, 2007.

[15] M. Camar-Eddine and P. Seppecher. Closure of the set of diffusion functionals with respect to the Mosco-convergence. Mathematical Models and Methods in Applied Sciences, 12(08):1153$1176,2002$. 
[16] M. Camar-Eddine and P. Seppecher. Determination of the closure of the set of elasticity functionals. Archive for Rational Mechanics and Analysis, 170(3):211-245, 2003.

[17] G. Dal Maso. An Introduction to Г-Convergence. Birkhuser, Boston, 1993.

[18] F. Dellisola, I. Giorgio, M. Pawlikowski, and N. Rizzi. Large deformations of planar extensible beams and pantographic lattices: Heuristic homogenization, experimental and numerical examples of equilibrium. Proceeding of the Royal Society of London, Sries A: Mathematical, Physical and Engineering sciences, 472(2185), 2016.

[19] B. Durand, A. Lebée, and S. Karam. Numerical study of pantographs. Personal communication, 2019.

[20] S. Gonella and M. Ruzzene. Homogenization and equivalent in-plane properties of twodimensional periodic lattices. International Journal of Solids and Structures, 45:2897-2915, 2008 .

[21] L. Jakabčin and P. Seppecher. On periodic homogenization of highly contrasted elastic structures. Hal preprint: hal-02379572, Nov. 2019.

[22] C. Pideri and P. Seppecher. A second gradient material resulting from the homogenization of an heterogeneous linear elastic medium. Continuum Mechanics and Thermodynamics, 9(5):241-257, 1997.

[23] E. Sanchez-Palencia. Non homogeneous media and vibration theory. Springer-Verlag, Berlin, 1980.

[24] P. Seppecher, J.-J. Alibert, and F. dellIsola. Linear elastic trusses leading to continua with exotic mechanical interactions. Journal of Physics: Conference Series, 319(1), 2011.

[25] V. P. Smyshlyaev and K. D. Cherednichenko. On rigorous derivation of strain gradient effects in the overall behaviour of periodic heterogeneous media. Journal of the Mechanics and Physics of Solids, 48:1325-1357, 2000. 